\title{
THE POSTPARTUM PESENTATION OF OSTEITIS PUBIS: A CASE REPORT
}

\author{
Ayça Uran Şan ${ }^{1}$, Müfit Akyüz ${ }^{1}$ H. Gülşah Karataş ${ }^{1}$, Ramazan Gündüz ${ }^{1}$ \\ ${ }^{1}$ Karabük University Karabük Training and Research Hospital, Department of Physical \\ Rehabilitation Medicine - Karabük, Turkey
}

Introduction: Osteitis pubis is characterised by painful and inflammatory condition of pubic smphysis. Even though, this pathology is usually associated with overuse injuries, pelvic surgery and trauma; it can be rarely seen in postpartum period.

Purpose: In this study we aimed to report a postpartum case of osteitis pubis who had admitted to our department with hip pain.

Method: A 28-year-old patient was referred to our hospital with bilateral mechanical hip pain. Her medical history revealed that she was in postpartum period and her pain had increased gradually after the parturition. On physical examination bilateral hip joint movements were painful. The strength of the right hip muscles were $3+/ 5$ and the left hip muscles were $4 / 5$.

Results: Routine laboratory tests revealed that the serum concentration of $C$ reactive protein (CRP) was $6.7 \mathrm{mg} / \mathrm{dl}$ and the erythrocyte sedimentation rate was $36 \mathrm{~mm} / \mathrm{h}$. A magnetic resonans imaging (MRI) was also planned for the patient. MRI findings showed subcortical and medullary bone marrow edema in the pubic bones bilaterally which were compatible with osteitis pubis. The patient has also referred to the department of gynecology. The gynecology department had detected a vaginal infection and applied drug theraphy to the patient. The conservative treatment included exercise programme was planned to the patient by our department. After the exercise programme VAS pain score was decreased from $100 \mathrm{~mm}$ to 60 $\mathrm{mm}$.
Discussion and Conclusion: Osteitis pubis occured in postpartum period is a rare clinical disorder. Clinicians should be aware of this clinical entity, especially in patients after the parturition. The diagnosis should be confirmed by clinical, labaratory and radiological examinations.

\section{REFERENCES:}

1. DeFinney J, Clements D, Staines M, Mior S. Osteitis pubis: a clinical challenge. The Journal of the Canadian Chiropractic Association. 1990;34(4):206-211.

2. Andrews SK, Carek PJ. Osteitis pubis: a diagnosis for the family physician.. J Am Board Fam Pract. 1998 JulAug;11(4):291-5

3. Khadabadi et al.. Postpartum Osteitis Pubis Following Spontaneous Vaginal Delivery: A Rare Cause of Pubalgia. International Journal of Orthopaedics Sciences 2015; 1(1): 16-18 